\title{
Protein levels, air pollution and vitamin D deficiency: links with
} \section{allergy}
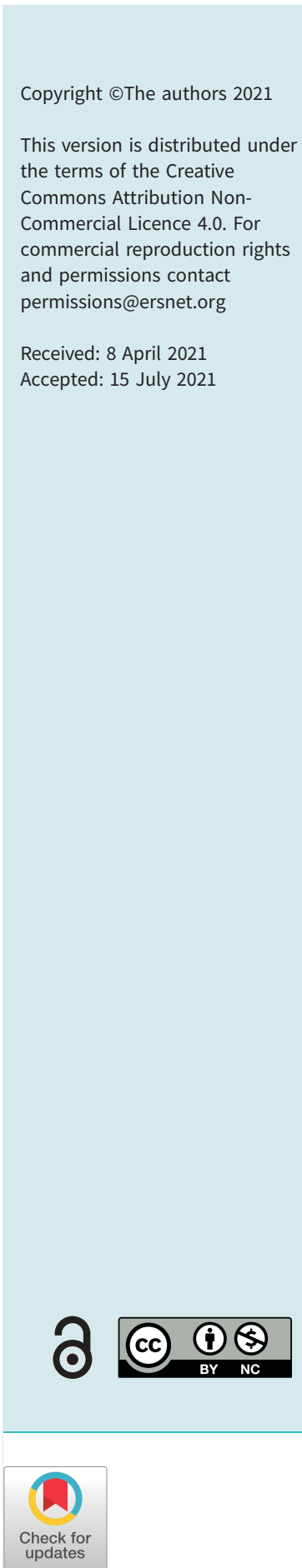

\section{To the Editor:}

The prevalence of allergic diseases has been increasing for several decades. This has been partly attributed to changing environmental factors such as exposure to traffic-related air pollution (TRAP) and nutrient deficiencies, including vitamin D. Furthermore, population-based studies have suggested that air pollution may contribute to vitamin D deficiency [1], while vitamin D levels may modify pollution-driven asthma symptoms in paediatric obesity [2]. Vitamin D supplementation has also been shown to reduce the effects of pollution on asthma and other chronic respiratory diseases [3]. To date, few mechanistic studies have aimed to identify the pathways that may explain these interactions.

Omic approaches are increasingly used to differentiate asthma and allergic disease endotypes, and to identify biomarkers and pathological mediators. To our knowledge, no study has assessed the proteomic response to more than one environmental factor, and their interactions, in the context of asthma and allergy. We therefore assessed the proteome in plasma from a subset of individuals in the Tasmanian Longitudinal Health Study (TAHS) cohort. We aimed to explore the effects of TRAP, using nitrogen dioxide $\left(\mathrm{NO}_{2}\right)$ as a proxy, and vitamin $\mathrm{D}$ status, on asthma and allergy and investigate the biological mechanisms that underlie these associations.

A subgroup ( $n=74)$ of middle-aged adults (mean \pm SD age $45 \pm 1$ years) was randomly selected from the 45 -year follow-up of the TAHS cohort [4]. As described previously [5], asthma and allergic conditions were defined by questionnaire and skin-prick tests respectively, while exposure to annual outdoor $\mathrm{NO}_{2}$ concentrations was estimated using a validated satellite-based land-use regression model approach [6]. We measured serum 25-hydroxyvitamin $\mathrm{D}_{3}\left(25(\mathrm{OH}) \mathrm{D}_{3}\right)$ levels using liquid chromatography-tandem mass spectrometry (LC-MS/MS) and analysed the proteomic profile using Orbitrap LC-MS/MS [7]. We categorised the participants into low or high $\mathrm{NO}_{2}$ exposure groups using a threshold of $5.1 \mathrm{ppb}$ based on the mean annual $\mathrm{NO}_{2}$ exposure of the TAHS cohort $(5.1 \pm 2.6 \mathrm{ppb}, \mathrm{n}=1367)$ [5] and we defined vitamin $\mathrm{D}$ deficiency as a $25(\mathrm{OH}) \mathrm{D}_{3}$ level $<50 \mathrm{nmol} \cdot \mathrm{L}^{-1}$ [8]. There were no differences in the sex distribution or smoking status between the low and high $\mathrm{NO}_{2}$ or between the vitamin $\mathrm{D}$ replete and deficient groups $(\mathrm{p}>0.05)$.

Air pollution influences the extent to which solar ultraviolet B radiation reaches the earth's surface and plays an independent role in the development of vitamin D deficiency in populations living in highly polluted areas [9]. However, in our cohort, where the $\mathrm{NO}_{2}$ levels were relatively low [5], we found no association between air pollution and vitamin $\mathrm{D}$ deficiency either using linear regression analysis $\left(\mathrm{r}=-0.091, \mathrm{p}=0.488\right.$ ) or by comparing the $\mathrm{NO}_{2}$ levels between the low and normal vitamin $\mathrm{D}$ groups ( $>0.05$ ) using independent t-tests. However, in line with our previous work [5], we did find an association between high $\mathrm{NO}_{2}$ levels and an increased risk of mixed grass (OR 6.85, 95\% CI 1.22-38.32; $\mathrm{p}=0.029$ ) and rye grass pollen allergy (OR 8.00, 95\% CI 1.56-40.99; $\mathrm{p}=0.013$ ) using logistic regression analyses with adjustment for sex and smoking status, but not with asthma or other allergic sensitisation phenotypes such as atopy and house dust mite sensitisation ( $p>0.05$ ). In contrast to $\mathrm{NO}_{2}$, vitamin $\mathrm{D}$ levels were not associated with allergic phenotypes in these middle-aged adults $(p>0.05)$, consistent with a previous finding that allergic sensitisation was associated with vitamin $\mathrm{D}$ deficiency in children and adolescents, but not adults [10]. Having established the main effect of outdoor air pollution on the onset of allergic diseases, we focussed subsequent analyses on exploring the biological mechanisms of air pollution induced allergy, considering vitamin $\mathrm{D}$ as a potential modifying factor.

\section{Shareable abstract (@ERSpublications)}

This study provides novel insights into mechanisms of traffic-related air pollution-induced allergy by down-regulation via complement regulators (CFI, PROS1 and PLG) and its interaction with vitamin D deficiency via the complement inhibitor PLG https://bit.ly/3x0jYOw

Cite this article as: Song Y, Walters EH, Abramson MJ, et al. Protein levels, air pollution and vitamin D deficiency: links with allergy. ERJ Open Res 2021; 7: 00237-2021 [DOI: 10.1183/23120541.00237-2021]. 
Of the 143 proteins quantified in blood proteomics, 13 were differentially expressed between the low and high exposure $\mathrm{NO}_{2}$ groups, including two (CD5L, $\mathrm{p}=0.016$; CETP, $\mathrm{p}=0.042$ ) upregulated and 11 (FCN3, $\mathrm{p}=0.020 ;$ PLG, $\mathrm{p}=0.022 ;$ IGKV1-17, $\mathrm{p}=0.042 ;$ IGKV1-39, $\mathrm{p}<0.001 ;$ IGLV2-11, $\mathrm{p}=0.023$; IGHV3-33, $\mathrm{p}=0.006$; IGKV1-16, $\mathrm{p}=0.021$; CFI, $\mathrm{p}=0.011$; PROS1, $\mathrm{p}=0.039 ;$ CFL1, $\mathrm{p}=0.037$; ACTG1, $\mathrm{p}=0.004$ ) downregulated in response to high $\mathrm{NO}_{2}$, with two (PLG and ACTG1) modified by vitamin D levels (interaction $\mathrm{p}=0.038$ and $\mathrm{p}=0.007$ respectively). Stratified analysis of these two proteins by vitamin $\mathrm{D}$ status showed that the significant differences were only present in the group with vitamin D deficiency (PLG: mean 0.228, 95\% CI $-0.287-0.743$ for low $\mathrm{NO}_{2}$ versus mean $-1.267,95 \% \mathrm{CI}-2.931-0.397$ for high $\mathrm{NO}_{2}, \mathrm{p}=0.013$; ACTG1: mean 0.084 , 95\% CI $-0.429-0.597$ for low $\mathrm{NO}_{2}$ versus mean $-1.794,95 \%$ CI $-3.341--0.246$ for high $\mathrm{NO}_{2}, \mathrm{p}=0.002$ ). We also identified 23 dysregulated proteins in the subjects with low vitamin D levels ( $\mathrm{p}<0.05$ ), of which five (IGKV1-17, IGLV2-11, IGHV3-33, IGKV1-16 and ACTG1) overlapped with proteins linked to $\mathrm{NO}_{2}$ exposure. These overlapping proteins were downregulated in the participants exposed to high $\mathrm{NO}_{2}$ and with low vitamin $\mathrm{D}$ (figure 1a).

Of the overlapping proteins, IGKV1-17 expression level was lower in the participants with asthma (mean -0.586 , 95\% CI -1.175-0.002) than those without asthma (mean 0.082, 95\% CI -0.183-0.347) ( $p=0.042$ ). However, we did not find any link between these proteins and other allergic outcomes. Most of the proteins identified as being differentially expressed are linked to immune regulation, complement activation, phagocytosis or proteolysis (figure 1b). Dysregulation of these proteins may represent common mechanisms through which air pollution and vitamin D deficiency drive adverse health outcomes. Further

a)
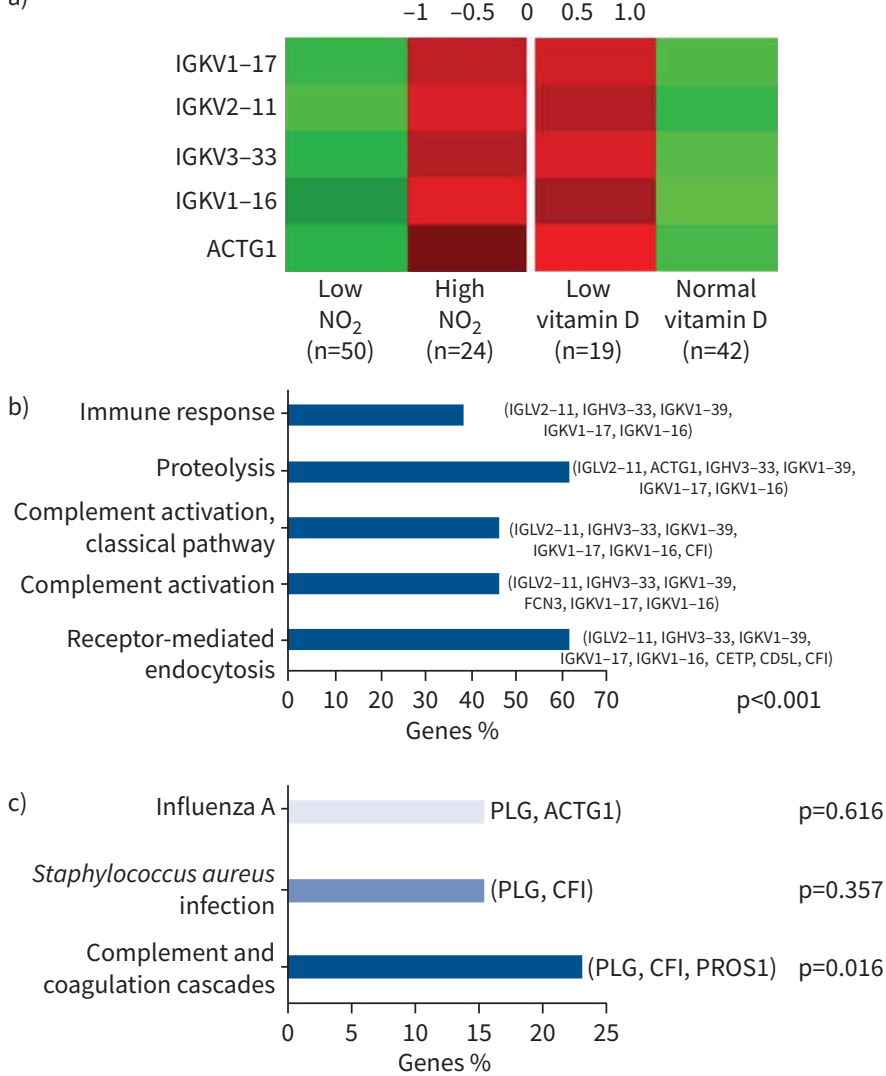
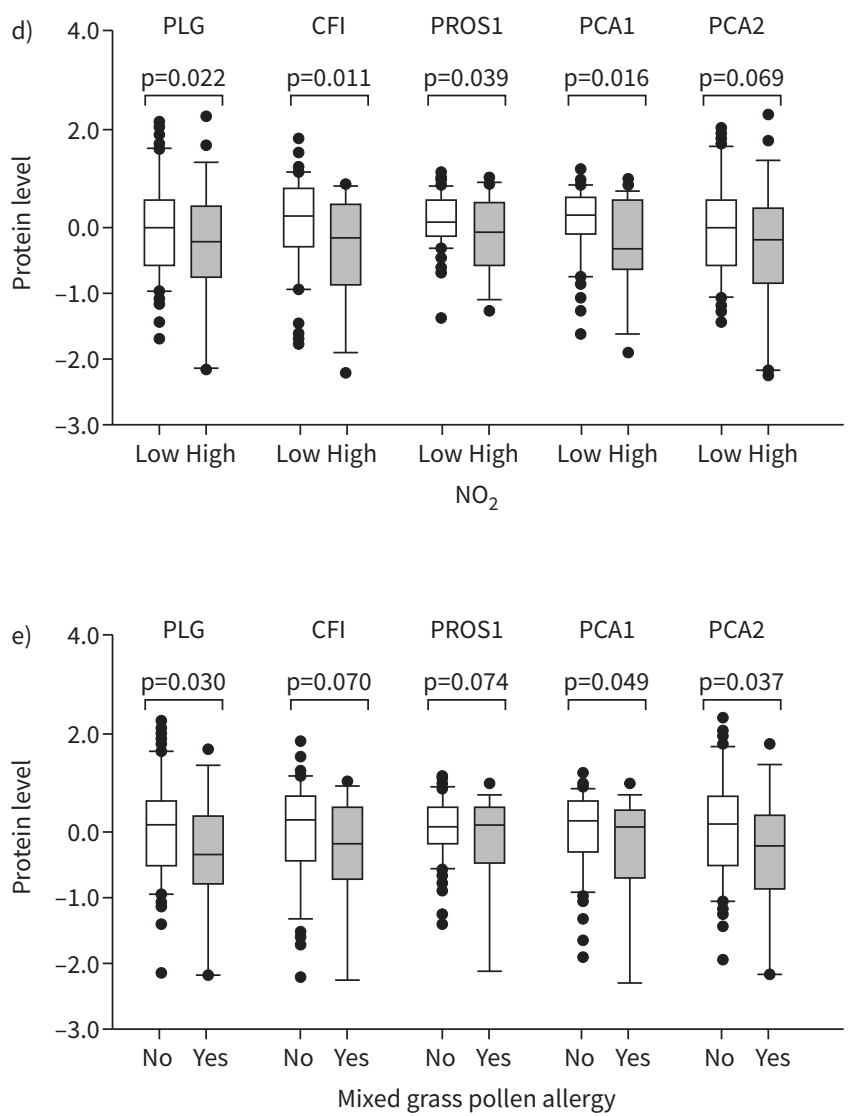

FIGURE 1 a) Heatmap showing expression of the five proteins downregulated in the participants with high $\mathrm{NO}_{2}$ and low vitamin D levels. b) Gene ontology annotation and c) the Kyoto Encyclopedia of Genes and Genomes (KEGG) database was used to identify significantly enriched biological processes and pathways, with the Benjamini $p$-value shown. The expression of selected proteins were compared between $d)$ low ( $n=50)$ and high $\mathrm{NO}_{2}(\mathrm{n}=24)$ groups, and e) in the individuals with $(\mathrm{n}=16)$ and without $(\mathrm{n}=54)$ mixed grass pollen (Kentucky bluegrass, orchard, redtop, timothy, sweet vernal grass and meadow fescue) allergy using general linear models, with adjustment for sex and smoking status. Principal component analysis (PCA) was applied to group the correlated proteins in the complement and coagulation cascades, with generation of two scores: PCA1 (56\%) and PCA2 (35\%). PLG: plasminogen; CFI: complement factor I; PROS1: protein S (alpha). 
investigation using a larger sample, including more diverse disease phenotypes, is warranted to link these findings directly to specific phenotypes.

Mapping of the 13 differentially expressed proteins associated with increased $\mathrm{NO}_{2}$ exposure revealed links to the complement and coagulation cascades (CCC) pathway (figure 1c). This was characterised by downregulation of three proteins, CFI, PLG and PROS1 (figure 1d), with one of these (PLG) modified by vitamin $\mathrm{D}$ deficiency. These proteins were also decreased in the participants with atopic responses to mixed grass pollen allergens (figure 1e), indicating an important potential role for these proteins in environmental (TRAP-related) allergy. Uncontrolled activation of CCC is thought to promote the development of Th2-driven allergic inflammation [11], although the exact mechanism is complex and remains elusive.

Given the inhibitory role of these proteins (CFI, PLG and PROS1) in targeting the central components of the C3 and C5 complement cascade, they may act in a coordinated way to activate CCC. For example, CFI is a serine protease which acts as a $\mathrm{C3b} / \mathrm{C} 4 \mathrm{~b}$ inactivator, while $\mathrm{C} 3 \mathrm{~b}$ is part of the $\mathrm{C} 5$ convertases that cleave C5 into anaphylatoxins (C5a) to amplify mast cell degranulation, contributing to increased Type I hypersensitivity $[11,12]$. PLG is a complement inhibitor that binds C3, the C3 cleavage products C3b and C3d, and C5, and enhances CFI-mediated C3b degradation [13]. PROS1 is a vitamin K-dependent glycoprotein cofactor to the serine protease that binds to the nascent complement complex C5,6,7 and prevents inappropriate activation of the complement system [14]. From a mechanistic perspective, air pollutants cause oxidative injury to the airways, leading to inflammation, remodelling and increased risk of sensitisation [15], and some studies suggest that oxidative stress mediates complement activation [16]. Therefore, it is of interest to determine whether the identified proteins are regulated by oxidative stress in the context of air pollution and allergy.

Thus, acknowledging the limitation of our sample size, we were able to identify three complement regulators that suggest a novel mechanism via activation of the complement system and subsequent development of allergy as a result of exposure to TRAP. Additionally, we were able to identify a potential mechanistic interaction between air pollution and vitamin D via the complement inhibitor, PLG. These data highlight the importance of considering interactions between multiple environmental exposures in the context of allergic diseases. Larger-scale molecular studies are warranted to externally validate and further investigate these interactions.

Yong Song ${ }^{1}$, E. Haydn Walters ${ }^{2,3}$, Michael J. Abramson $\oplus^{4}$, Gayan Bowatte ${ }^{2,5,6}$, Dinh S. Bui ${ }^{2}$, Jennifer Perret $\oplus^{2}$, lain Feather ${ }^{7}$, Luke D. Knibbs ${ }^{8}$, Richard Wilson ${ }^{9}$, David S. Nichols ${ }^{9}$, Shyamali C. Dharmage $\mathbb{\oplus}^{2}$ and Graeme R. Zosky ${ }^{1,3}$

${ }^{1}$ Menzies Institute for Medical Research, College of Health and Medicine, University of Tasmania, Hobart, Tasmania, Australia. ${ }^{2}$ School of Population and Global Health, The University of Melbourne, Carlton, Victoria, Australia. ${ }^{3}$ Tasmanian School of Medicine, University of Tasmania, Hobart, Tasmania, Australia. ${ }^{4}$ School of Public Health and Preventive Medicine, Monash University, Melbourne, Victoria, Australia. ${ }^{5}$ Faculty of Allied Health Sciences, University of Peradeniya, National Institute of Fundamental Studies, Kandy, Sri Lanka. ${ }^{6}$ Department of Basic Sciences, Faculty of Allied Health Sciences, University of Peradeniya, Kandy, Sri Lanka. ${ }^{7}$ Gold Coast Hospital, Southport, Queensland, Australia. ${ }^{8}$ School of Public Health, The University of Queensland, Herston, Queensland, Australia. ${ }^{9}$ Central Science Laboratory, University of Tasmania, Hobart, Tasmania, Australia.

Corresponding author: Shyamali C. Dharmage (s.dharmage@unimelb.edu.au)

Provenance: Submitted article, peer reviewed.

Acknowledgments: We acknowledge the TAHS study participants and previous investigators. We thank Prof. Mark Jenkins, Centre for Epidemiology and Biostatistics, The University of Melbourne, Victoria, a TAHS investigator, but not a coauthor of this manuscript, for his assistance with obtaining funds and data collection. We also acknowledge all the respiratory scientists who collected data in the lung function laboratories of Tasmania, Victoria, Queensland, South Australia, Western Australia and New South Wales; and the research interviewers, data entry operators and research officers. We thank the Archives Office of Tasmania for providing data from the 1968 TAHS questionnaires. Finally, we also thank all participants in ECLIPSE for their willingness to contribute to medical research, as well all investigators involved in the study. 
Conflict of interest: M.J. Abramson holds investigator-initiated grants for unrelated research from Pfizer and Boehringer Ingelheim. He has undertaken an unrelated consultancy for and received assistance with conference travel from Sanofi. He has also received a speaker's fee from GSK. S.C. Dharmage holds investigator-initiated grants for unrelated research from GSK. The other authors declare that they have no relevant conflicts of interest.

Support statement: Tasmanian Longitudinal Health Study was supported by the National Health and Medical Research Council (NHMRC) of Australia under the NHMRC project grant scheme (299901 and 1021275) and NHMRC European collaborative grant scheme (1101313) as part of ALEC (Ageing Lungs in European Cohorts) (funded by the European Union's Horizon 2020 research and innovation programme under grant agreement number 633212); The University of Melbourne; Clifford Craig Medical Research Trust of Tasmania; the Victorian, Queensland and Tasmanian Asthma Foundations; The Royal Hobart Hospital; Helen MacPherson Smith Trust; and GlaxoSmithKline. S.C. Dharmage, J. Perret and E.H. Walters are supported by the NHMRC of Australia. Funding information for this article has been deposited with the Crossref Funder Registry.

\section{References}

1 Hosseinpanah $\mathrm{F}$, Pour SH, Heibatollahi M, et al. The effects of air pollution on vitamin D status in healthy women: a cross sectional study. BMC Public Health 2010; 10: 519.

2 Barrea L, Savastano S, Di Somma C, et al. Low serum vitamin D-status, air pollution and obesity: a dangerous liaison. Rev Endocr Metab Disord 2017; 18: 207-214.

3 Whyand $\mathrm{T}$, Hurst JR, Beckles $\mathrm{M}$, et al. Pollution and respiratory disease: can diet or supplements help? A review. Respir Res 2018; 19: 79.

4 Matheson MC, Abramson MJ, Allen K, et al. Cohort profile: the Tasmanian Longitudinal Health Study (TAHS). Int J Epidemiol 2017; 46: 407-408i.

5 Bowatte G, Lodge CJ, Knibbs LD, et al. Traffic-related air pollution exposure is associated with allergic sensitization, asthma, and poor lung function in middle age. J Allergy Clin Immunol 2017; 139: 122-129.

6 Knibbs LD, Hewson MG, Bechle MJ, et al. A national satellite-based land-use regression model for air pollution exposure assessment in Australia. Environ Res 2014; 135: 204-211.

7 Yen S, Song $\mathrm{Y}$, Preissner $\mathrm{M}$, et al. The proteomic response is linked to regional lung volumes in ventilator-induced lung injury. J Appl Physiol (1985) 2020; 129: 837-845.

$8 \quad$ Holick MF. Vitamin D deficiency. N Engl J Med 2007; 357: 266-281.

9 Mousavi SE, Amini H, Heydarpour P, et al. Air pollution, environmental chemicals, and smoking may trigger vitamin D deficiency: evidence and potential mechanisms. Environ Int 2019; 122: 67-90.

10 Sharief S, Jariwala S, Kumar J, et al. Vitamin D levels and food and environmental allergies in the United States: results from the National Health and Nutrition Examination Survey 2005-2006. J Allergy Clin Immunol 2011; 127: 1195-1202.

11 Zhang X, Kohl J. A complex role for complement in allergic asthma. Expert Rev Clin Immunol 2010; 6: 269-277.

12 Merle NS, Church SE, Fremeaux-Bacchi V, et al. Complement system part I - molecular mechanisms of activation and regulation. Front Immunol 2015; 6: 262.

13 Barthel D, Schindler S, Zipfel PF. Plasminogen is a complement inhibitor. J Biol Chem 2012; 287: 18831-18842.

14 Milis L, Morris CA, Sheehan MC, et al. Vitronectin-mediated inhibition of complement: evidence for different binding sites for C5b-7 and C9. Clin Exp Immunol 1993; 92: 114-119.

15 Guarnieri M, Balmes JR. Outdoor air pollution and asthma. Lancet 2014; 383: 1581-1592.

16 Collard CD, Vakeva A, Morrissey MA, et al. Complement activation after oxidative stress: role of the lectin complement pathway. Am J Pathol 2000; 156: 1549-1556. 